\title{
Application of a multi-field synergy principle in the performance evaluation of convective heat transfer enhancement in a tube
}

\author{
LIU Wei", LIU ZhiChun \& MA Lei \\ School of Energy and Power Engineering, Huazhong University of Science and Technology, Wuhan 430074, China
}

Received October 11, 2011; accepted January 10, 2012

\begin{abstract}
For laminar and turbulent convective heat transfer, the synergy among vectorial physical quantities of a fluid particle is analyzed to reveal the relation between the multi-field synergy mechanism and heat transfer enhancement. Additionally, the efficiency evaluation criterion (EEC) is proposed to evaluate the overall performance of heat transfer enhancement. Meanwhile, using synergy angles $\alpha, \beta, \theta, \gamma$ and $\eta$, a unified evaluation system and corresponding evaluation indexes for heat transfer enhancement are suggested. A model of a heat-transfer-enhanced tube inserted with poles in a triangular configuration is built, and a corresponding numerical simulation is conducted to verify the proposed evaluation system and criterion. The calculation results show that there is correlation between synergy angles reflecting the direction of heat transfer enhancement and evaluation criterion reflecting the effect of heat transfer enhancement. In the Re number range of 300-1800, the performance evaluation criterion PEC lies in the range of 1.2-2.3, but the efficiency evaluation criterion EEC lies in the range of 0.33-0.45.
\end{abstract}

multi-field synergy, heat-transfer-enhanced tube, laminar flow, turbulent flow, performance evaluation

Citation: Liu W, Liu Z C, Ma L. Application of a multi-field synergy principle in the performance evaluation of convective heat transfer enhancement in a tube. Chin Sci Bull, 2012, 57: 1600-1607, doi: 10.1007/s11434-012-5062-x

To make heat exchangers more efficient, the most important thing is to raise the heat transfer coefficient and reduce the fluid power consumption in the transport processes. Additionally, designing heat exchangers with small size and low weight is important to save energy. Therefore, the problem is how to improve the comprehensive performance of a heat exchanger by reconciling heat resistance with flow resistance. For almost half a century, various approaches of heat transfer enhancement had been developed and applied to improve the performance of heat exchangers. Researchers have made efforts to classify such approaches; e.g., Webb [1] classified the approaches as active enhancement, passive enhancement and composite enhancement, and Bergles [2] described the evolution of those approaches in four generations. These classifications, however, did not relate to the mechanism of enhancing heat transfer and reducing flow resistance, and did not involve any evaluation standard. Guo et al. [3] provided a new insight into the physical mecha-

*Corresponding author (email: w_liu@ @ust.edu.cn) nism of convective heat transfer by analyzing the relation between flow field and heat flux field. Considering that the heat transfer can be related to distribution characteristics of the velocity field and temperature field, Guo et al. proposed the field synergy principle of heat transfer enhancement. Verified in a series of experiments and numerical simulations [4-21], the principle provided a theoretical foundation for evaluating the performance of heat transfer enhancement.

\section{Performance evaluation for heat transfer enhancement}

A problem we face in developing approaches to enhance heat transfer is that heat transfer is enhanced at the cost of an undesirable increase in flow resistance. Sometimes the energy saved by enhancing heat transfer may be less than the increase in energy cost due to flow resistance. Therefore, an objective evaluation of the effect of heat transfer enhancement is necessary in designing high-efficiency and 
low-resistance heat transfer units and exchangers.

\subsection{Synergy based on the momentum and energy equa- tions}

The momentum equation for laminar convective heat transfer is

$$
-\nabla p=\rho \boldsymbol{U} \cdot \nabla \boldsymbol{U}-\mu \nabla^{2} \boldsymbol{U},
$$

and that for turbulent convective heat transfer is

$$
-\nabla p=\rho \boldsymbol{U} \cdot \nabla \boldsymbol{U}-\rho\left(v+\varepsilon_{m}\right) \nabla^{2} \boldsymbol{U},
$$

where $\rho$ is fluid density, $\mu$ is fluid viscosity, $v$ is molecular momentum diffusivity, and $\varepsilon_{m}$ is turbulent momentum diffusivity.

Using eqs. (1) and (2), refs. [19-21] established a twodimensional momentum synergy equation for laminar flow,

$$
E u_{l}=\frac{0.457 \chi_{1}}{\left(\Lambda R e_{l}\right)^{0.5}}+\frac{3 \chi_{2}}{R e_{l}}+\int_{0}^{1} \int_{0}^{\delta / h}(\bar{U} \cdot \nabla \bar{u}) \mathrm{d} X \mathrm{~d} Y,
$$

and a two-dimensional momentum synergy equation for turbulent flow,

$$
E u_{t}=\frac{0.021 \chi_{1}}{\left(\Lambda R e_{t}\right)^{0.2}}+\frac{0.079 \chi_{2}}{R e_{t}^{0.25}}+\int_{0}^{1} \int_{0}^{\delta / h}(\overline{\boldsymbol{U}} \cdot \nabla \bar{u}) \mathrm{d} X \mathrm{~d} Y,
$$

where $E u_{l}$ and $E u_{t}$ are the Euler numbers for laminar flow and turbulent flow respectively, $E u=\frac{\Delta p}{\rho u_{m}^{2}} ; R e_{l}$ and $R e_{t}$ are Reynolds number for laminar flow and turbulent flow respectively; $\chi_{1}$ and $\chi_{2}$ are the ratios of the entrance region and fully developed region of the channel to the total channel length respectively; $\Lambda$ is the ratio of the entrance length to channel height; and $\bar{U}$ and $\bar{u}$ are the dimensionless velocity vector and velocity. The first and second terms on the right hand side of eqs. (3) and (4) are flow resistances in the entrance region and fully developed region respectively. The dot product of the dimensionless velocity vector and velocity gradient can be expressed both for laminar flow and turbulent flow as

$$
\overline{\boldsymbol{U}} \cdot \nabla \bar{u}=|\overline{\boldsymbol{U}}||\nabla \bar{u}| \cos \alpha .
$$

From eqs. (3)-(5), it is found that the larger the angle $\alpha$ between vectors $\boldsymbol{U}$ and $\nabla u$, the smaller the dot product $\overline{\boldsymbol{U}} \cdot \nabla \bar{u}$ and thus the smaller the Euler numbers of laminar flow and turbulent flow. Therefore, increasing the angle $\alpha$ will reduce the pressure drop. Here the synergy angle $\alpha$ is universally expressed as

$$
\alpha=\arccos \frac{\boldsymbol{U} \cdot \nabla u}{|\boldsymbol{U}||\nabla u|} .
$$

For a heat exchanger, when power consumption is constant, less flow resistance results in higher fluid velocity, which implies better heat transfer. In this case, there are two aspects to the improvement in the performance of the heat exchanger: the reduction of flow resistance and the enhancement of heat transfer. However, when the fluid is accelerated by increasing pump power, not only does convec- tive heat transfer between the fluid and the solid wall strengthens, but the flow resistance increases as well. Therefore, enhancing convective heat transfer by merely increasing the fluid velocity or reducing flow resistance by merely decreasing the fluid velocity does not make sense as an approach to improve the comprehensive performance of a heat transfer unit. Thus, when a heat transfer unit is to be evaluated in terms of its performance of heat transfer enhancement, the fluid velocity could be assumed as being constant.

There are two cases to consider when $|\boldsymbol{U}|$ is constant. (1) If $|\nabla u|$ is constant, then the larger the synergy angle $\alpha$, the smaller the dot product $\boldsymbol{U} \cdot \nabla u$ and thus the smaller the $E u$ number. (2) If angle $\alpha$ is constant, then the smaller the $|\nabla u|$ value, the smaller the dot product $\boldsymbol{U} \cdot \nabla u$ and thus the smaller the $E u$ number. In addition, when $|\boldsymbol{U}|$ is constant, the smaller $|\nabla u|$ is, the smaller $\nabla p$ will be. As a result, the flow resistance of fluid will be lower.

For laminar convective heat transfer, the energy equation is

$$
-\nabla \cdot \boldsymbol{q}=\rho c \boldsymbol{U} \cdot \nabla T,
$$

and for turbulent convective heat transfer, the energy equation is

$$
\left(\lambda+\rho c_{p} \varepsilon_{h}\right) \nabla^{2} T=\rho c_{p} \boldsymbol{U} \cdot \nabla T,
$$

where $\lambda$ is fluid conductivity, $c_{p}$ is the specific heat of the fluid, and $\varepsilon_{h}$ is turbulent thermal diffusivity.

From eqs. (7) and (8), refs. [3] and [19-21] established a two-dimensional energy synergy equation for laminar flow,

$$
N u_{l}=\operatorname{Re}_{l} \operatorname{Pr}_{l} \int_{0}^{\delta_{t} / h}(\overline{\boldsymbol{U}} \cdot \nabla \bar{T}) \mathrm{d} Y,
$$

and a two-dimensional energy synergy equation for turbulent flow,

$$
N u_{t}=\operatorname{Re}_{t} \operatorname{Pr}_{t} \int_{0}^{\delta_{t} / h}(\bar{U} \cdot \nabla \bar{T}) \mathrm{d} Y,
$$

where $N u_{l}, N u_{t}, R e_{l}, R e_{t}, P r_{l}$ and $P r_{t}$ represent the Nusselt number, Reynolds number and Prandtl number for laminar flow and turbulent flow respectively, and $\nabla \bar{T}$ is a dimensionless temperature gradient. The dot product of the dimensionless velocity vector and temperature gradient can be expressed both for laminar flow and turbulent flow as

$$
\overline{\boldsymbol{U}} \cdot \nabla \bar{T}=|\overline{\boldsymbol{U}}||\nabla \bar{T}| \cos \beta
$$

From eqs. (9)-(11), it is found that the smaller the angle $\beta$ between vectors $\boldsymbol{U}$ and $\nabla T$, the larger the dot product $\bar{U} \cdot \nabla \bar{T}$ and thus the larger the $N u$ number. Thus, convective heat transfer between the fluid and solid wall will be enhanced. Here the synergy angle $\beta$ can be universally expressed as

$$
\beta=\arccos \frac{\boldsymbol{U} \cdot \nabla T}{|\boldsymbol{U}||\nabla T|}
$$


As before, there are two cases to consider when $|\boldsymbol{U}|$ is constant. (1) If $|\nabla T|$ is constant, then the smaller the angle $\beta$, the larger the dot product $\boldsymbol{U} \cdot \nabla T$ and thus the larger the $N u$ number. (2) If angle $\beta$ is constant, then the larger the $|\nabla T|$ value, the larger the dot product $U \cdot \nabla T$ and thus the larger the $\mathrm{Nu}$ number. As a result, the convective heat transfer between the fluid and tube wall increases.

In the situation of heat transfer enhancement in a tube, there is a main-flow direction along the axis of tube. Therefore, $\nabla u$ can be taken as a reference vector, and vectorial physical quantities of the fluid particle $M$ can be represented as a tetrahedron volume described by synergy angles $\alpha, \beta$ and $\gamma$, as shown in Figure 1.

To assess the heat transfer performance of heat transfer units, refs. [22,23] defined a comprehensive evaluation index:

$$
\begin{aligned}
& \text { PEC }=\frac{h A / h_{0} A_{0}}{\left(P / P_{0}\right)^{1 / 3}\left(A / A_{0}\right)^{2 / 3}}= \\
& \frac{N u / N u_{0}}{\left(f / f_{0}\right)^{1 / 3}\left(R e / R e_{0}\right)\left(P r / P r_{0}\right)^{1 / 3}},
\end{aligned}
$$

where $\mathrm{Nu}, \mathrm{Re}$ and $\mathrm{Pr}$ denote the Nusselt number, Reynolds number and Prandtl number of laminar flow or turbulent flow; $P$ is the power consumption of the fluid; $h$ is the coefficient of convective heat transfer; $f$ is the resistance coefficient; and $A$ is the area of heat transfer. With the same Re number, when physical properties of the fluid remain unchanged and the area of heat transfer area is fixed, eq. (13) can be rewritten for the same power consumption as

$$
\mathrm{PEC}=\frac{N u / N u_{0}}{\left(f / f_{0}\right)^{1 / 3}},
$$

where $N u_{0}$ and $f_{0}$ are the Nusselt number and resistance coefficient of a bare tube respectively.

From the above analysis, it is seen that the synergy relation given in Figure 1 is related to the performance evaluation criterion in eq. (14). If the objective is to enhance heat

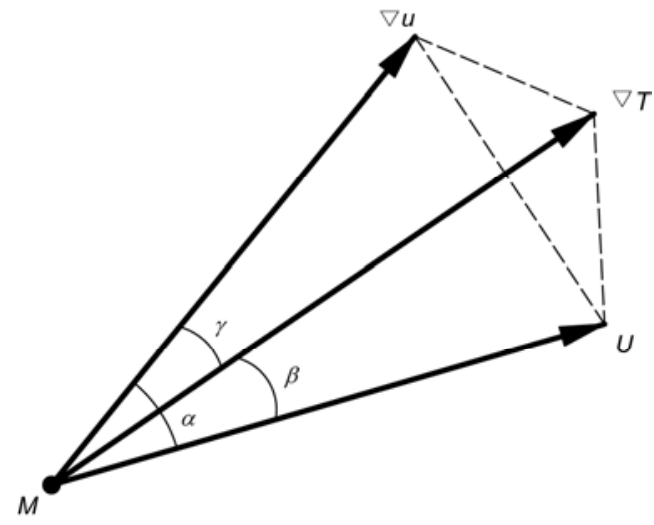

Figure 1 Synergy relations among the velocity vector, velocity gradient and temperature gradient of a fluid particle $M$. transfer, the synergy between the velocity vector $\boldsymbol{U}$ and temperature gradient $\nabla T$ should be considered, and the smaller the synergy angle $\beta$ is, the more effective the convective heat transfer will be. As a result, $N u / N u_{0}$ and PEC will increase. If the objective is to achieve minimum flow resistance, the synergy between the velocity vector $\boldsymbol{U}$ and velocity gradient $\nabla u$ should be considered, and the larger the synergy angle $\alpha$ is, the smaller the momentum loss will be. As a result, $\left(f / f_{0}\right)^{1 / 3}$ will decrease and PEC will increase. In addition, the smaller $|\nabla u|$ is, the smaller the flow resistance will be; and the larger $|\nabla T|$ in the boundary layer is, the more effective the convective heat transfer will be.

\subsection{Synergy based on the conservation equation for mechanical energy}

Multiplying both sides of eq. (1) by the velocity vector $\boldsymbol{U}$ yields

$$
-\nabla p \cdot \boldsymbol{U}=\left(\rho \boldsymbol{U} \cdot \nabla \boldsymbol{U}-\mu \nabla^{2} \boldsymbol{U}\right) \cdot \boldsymbol{U},
$$

and multiplying both sides of eq. (2) by the velocity vector $\boldsymbol{U}$ yields

$$
-\nabla p \cdot \boldsymbol{U}=\left(\rho \boldsymbol{U} \cdot \nabla \boldsymbol{U}-\rho\left(v+\varepsilon_{m}\right) \nabla^{2} \boldsymbol{U}\right) \cdot \boldsymbol{U} .
$$

The vector mode equations of the conservation of mechanical energy for laminar flow and turbulent flow can be expressed as

$$
|-\nabla p||\boldsymbol{U}|=\left|\rho \boldsymbol{U} \cdot \nabla \boldsymbol{U}-\mu \nabla^{2} \boldsymbol{U}\right||\boldsymbol{U}|,
$$

and

$$
|-\nabla p||\boldsymbol{U}|=\left|\rho \boldsymbol{U} \cdot \nabla \boldsymbol{U}-\rho\left(v+\varepsilon_{m}\right) \nabla^{2} \boldsymbol{U}\right||\boldsymbol{U}|,
$$

where $-\nabla p \cdot \boldsymbol{U}$ is the rate at which work is done by the fluid and $|-\nabla p||\boldsymbol{U}|$ is pump work consumed by the fluid.

Eqs. (15) and (16) describe the conservation relation of the mechanical energy of fluid, which states that the work consumed by the fluid equals the sum of kinetic energy loss and viscous dissipation work. Eqs. (17) and (18) show that less power consumption means lower kinetic energy and viscous dissipation losses.

From the mechanical energy conservation expressed in the above equations and the relation among vectorial physical quantities of a fluid particle $M$, we can obtain the synergy relation between velocity vector $\boldsymbol{U}$ and pressure gradient $\nabla p$ both for laminar flow and turbulent flow as

$$
\boldsymbol{U} \cdot(-\nabla p)=|\boldsymbol{U}||-\nabla p| \cos \theta
$$

or

$$
\theta=\arccos \frac{\boldsymbol{U} \cdot(-\nabla p)}{|\boldsymbol{U}||-\nabla p|} .
$$

From eqs. (17)-(20), one finds that (1) if the dot product $\boldsymbol{U} \cdot(-\nabla p)$ is fixed, then the smaller the angle $\theta$ is, the less 
the power consumption $|\boldsymbol{U}||-\nabla p|$ will be, and (2) if the angle $\theta$ is fixed, then the smaller the dot product $\boldsymbol{U} \cdot(-\nabla p)$ is, the less the power consumption $|\boldsymbol{U}||-\nabla p|$ will be. Thus, reducing synergy angle $\theta$ means consuming less pump power, which increases the energy savings for heat transfer units.

The vector tetrahedral volume in Figure 2 shows that if the angle $\eta$ is fixed, then the smaller the angle $\beta$ is, the larger the angle $\theta$ will be; or the smaller the angle $\theta$ is, the larger the angle $\beta$ will be. Therefore, the objective of intensifying heat transfer opposes that of reducing flow resistance. When the heat transfer is enhanced, the flow resistance increases; or when the flow resistance decreases, the heat transfer will weaken. Obviously, there must be a certain optimization relation among synergy angles $\beta, \theta$ and $\eta$.

If heat transfer performance is evaluated according to fluid power consumption, we can define efficiency evaluation criterion as

$$
\mathrm{EEC}=\frac{Q / Q_{0}}{P / P_{0}}=\frac{Q / Q_{0}}{(V \Delta p) /\left(V_{0} \Delta p_{0}\right)},
$$

where the numerator represents the heat flux income, the denominator represents the power consumption cost, $Q$ is heat flux, $P$ is power consumption, and $V$ is the volume flow rate of the fluid. The subscript 0 indicates the bare tube. When the flow rate remains unchanged, eq. (21) can be written as

$$
\mathrm{EEC}=\frac{Q / Q_{0}}{\Delta p / \Delta p_{0}} .
$$

Compared with PEC, EEC clearly represents the comprehensive performance of a heat transfer unit. When the fluid's physical properties and the area of heat transfer remain unchanged, eq. (22) becomes

$$
\begin{aligned}
& \mathrm{EEC}=\frac{\left(h / h_{0}\right)\left(\Delta T / \Delta T_{0}\right)}{\Delta p / \Delta p_{0}} \approx \\
& \zeta \frac{N u / N u_{0}}{f / f_{0}} \approx \frac{N u / N u_{0}}{f / f_{0}},
\end{aligned}
$$

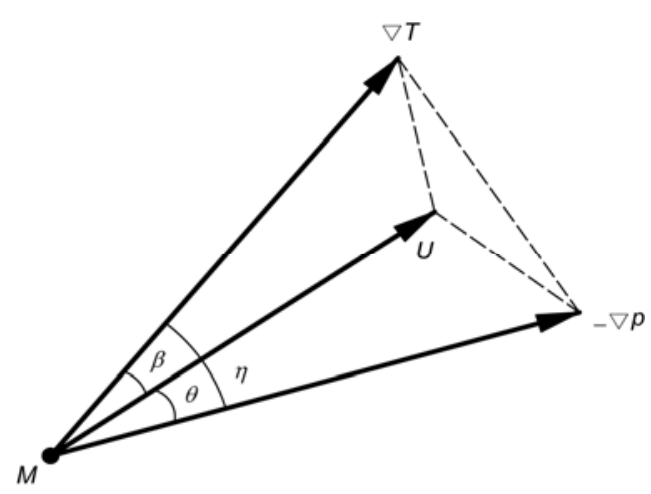

Figure 2 Synergy relation among the velocity vector, temperature gradient and pressure gradient of a fluid particle $M$. where $\Delta T=T_{w}-T_{f}$ is the difference between the tube wall temperature and fluid mean temperature, $\Delta T_{0}=$ $T_{w 0}-T_{f 0}$ is the temperature difference for the bare tube, and $\zeta=\Delta T / \Delta T_{0}$ is a factor of the temperature difference. Therefore, EEC not only represents the difference in the heat transfer ability and the characteristic of flow resistance between the heat-transfer-enhanced tube and bare tube, but it also considers the effect of temperature variation on heat transfer.

Figure 2 reflects the synergy relation in the flow field of convective heat transfer, which is closely related to the evaluation criterion defined in eq. (22). For example, the smaller the angle $\beta$ is, the larger the ratio of $Q / Q_{0}$ will be; while the smaller the angle $\theta$ is, the smaller the ratio of $\Delta p / \Delta p_{0}$ will be. This will lead to a higher EEC value. Therefore, the performance of heat transfer enhancement can be improved by reducing synergy angles $\beta$ and $\theta$. In addition, in the system constructed in Figure 2, the smaller $|-\nabla p|$ is, the smaller the flow resistance will be; and the larger $|\nabla T|$ in the boundary layer is, the stronger the convective heat transfer between the fluid and solid wall will be.

The above analysis shows that the angles $\beta$ and $\theta$ formed by vectors $\boldsymbol{U}, \nabla T$ and $-\nabla p$ not only reflect the relation between heat transfer and flow, but also reveal the mechanism of enhancing heat transfer and reducing flow resistance, which is beneficial to achieve the ultimate goal of improving the comprehensive performance of a heat transfer unit.

\subsection{Comprehensive evaluation indexes of heat transfer enhancement}

Following Figures 1 and 2, the synergy relations among all vectors are shown in Figure 3, in which the synergy angles formed by $\boldsymbol{U}, \nabla u, \nabla T$ and $-\nabla p$ reflect the mechanism of heat transfer enhancement, while the mode values $|\boldsymbol{U}|$, $|\nabla u|,|\nabla T|$ and $|-\nabla p|$ reflect the field intensity of convective heat transfer and are quantified by the Nusselt number, convective heat transfer coefficient, fluid resistance coefficient, heat transfer rate and pressure difference. Accordingly, we define a set of indexes to evaluate the performance of heat transfer enhancement; e.g., $\alpha, \beta, \theta, N u, f$, PEC, $Q, \Delta p$, and EEC. If comparing the heat-transferenhanced tube with the bare tube, we can take $\beta, \theta$ and EEC as basic indexes.

The relation between micro-element areas and vectors of a fluid particle is shown in Figure 4, in which $A$ represents the micro-element area of mass flow, with $\boldsymbol{U}$ as its normal vector; $B$ represents the micro-element area of driving potential, with $-\nabla p$ as its normal vector; and $C$ represents 


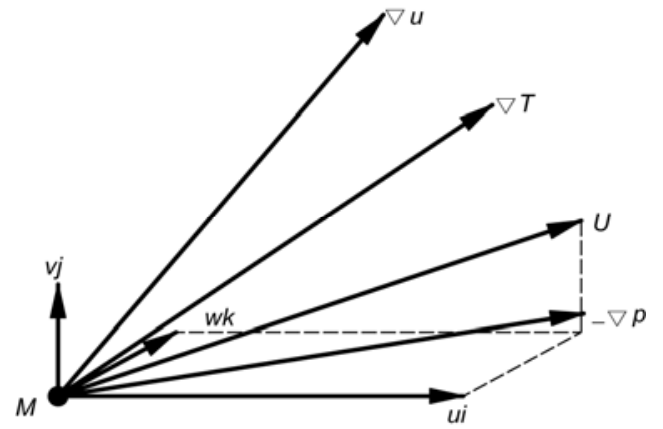

Figure 3 Three-dimensional synergy relation among vectors for a fluid particle $M$.

the micro-element area of heat flux, with $\nabla T$ as its normal vector. The fluid particle $M$ is located at the intersection of the three micro-element areas. Figure 4 shows that there exists competition between synergy angles. For a certain angle $\eta$, if vector $\boldsymbol{U}$ leans toward vector $\nabla T$, then synergy angle $\beta$ decreases, leading to a reduction in thermal resistance. If vector $\boldsymbol{U}$ leans toward vector $-\nabla p$, then synergy angle $\theta$ decreases, leading to a reduction in flow resistance. It is thus concluded that the comprehensive performance of heat transfer enhancement would be improved by optimizing synergy angles $\beta, \theta$ and $\eta$.

Figure 5 shows the framework of the multi-field synergy principle of heat transfer enhancement. In the figure, the dot product of $\boldsymbol{U}$ and $\nabla u$ from the momentum equation forms synergy angle $\alpha$; the dot product of $\boldsymbol{U}$ and $\nabla T$ from the energy equation forms synergy angle $\beta$; the dot product of $\boldsymbol{U}$ and $-\nabla p$ from the mechanical energy conservation equation forms synergy angle $\theta$, vectors $\nabla u$ and $\nabla T$ form synergy angle $\gamma$, and vectors $\nabla T$ and $-\nabla p$ form synergy angle $\eta$. With all these angles, the basic relation of the multi-field synergy principle is expressed, which reflects the regularity between heat transfer enhancement and synergy among the velocity field, temperature field and pressure field.

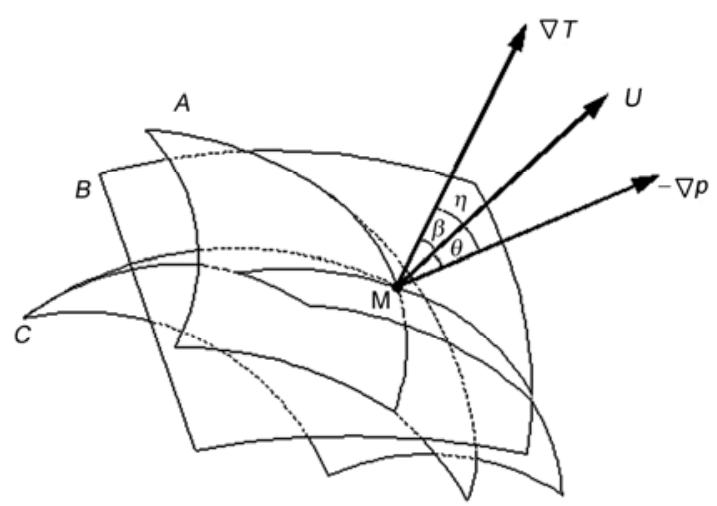

Figure 4 Relation among the micro-element areas and vectors for a fluid particle $M$ and micro-element areas of mass flow $(A)$, driving potential $(B)$, and heat flux $(C)$.

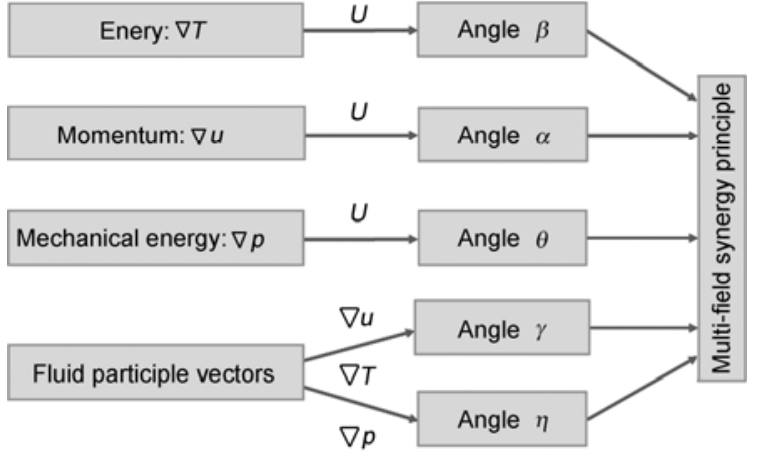

Figure 5 Framework of the multi-field synergy principle for convective heat transfer enhancement.

\section{Computation and analysis of heat transfer enhancement}

To verify the proposed evaluation indexes through numerical computation, we choose a model of a circular tube inserted with triangular arrangements of tiny poles as shown in Figure 6. The length of the tube is $500 \mathrm{~mm}$, the diameter of the tube is $20 \mathrm{~mm}$, and the diameter of a pole is $1 \mathrm{~mm}$. The triangular pole arrangements are themselves arranged in a staggered pattern with an angle of rotation of $60^{\circ}$ and an interval of $10 \mathrm{~mm}$. The space between the tube wall and the end of a triangular arrangement is $1 \mathrm{~mm}$.

A generalized governing equation for laminar convective heat transfer can be expressed as

$$
\begin{aligned}
& \frac{\partial(\rho u \Phi)}{\partial x}+\frac{\partial(\rho v \Phi)}{\partial y}+\frac{\partial(\rho w \Phi)}{\partial z} \\
& =\frac{\partial}{\partial x}\left(\Gamma \frac{\partial \Phi}{\partial x}\right)+\frac{\partial}{\partial y}\left(\Gamma \frac{\partial \Phi}{\partial y}\right)+\frac{\partial}{\partial z}\left(\Gamma \frac{\partial \Phi}{\partial z}\right)+S,
\end{aligned}
$$

where $\rho$ is fluid density; $u, v$ and $w$ are velocity components along the $x, y$ and $z$ directions; $\Gamma$ is generalized diffusivity [24]; $S$ is a source item; $\Phi$ is a universal variable, with $\Phi=1$ for the continuity equation; $\Phi=u, v, w$ for the momentum equations; and $\Phi=T$ for the energy equation.

The finite difference approach is adopted for numerical computation and the SIMPLE approach is used for pressure-velocity coupling. The difference scheme takes the second-order upwind difference, and the QUICK scheme is employed for the convection term. In the calculation, the tube wall temperature $T_{w}$ and fluid inlet temperature $T_{\infty}$ are set at 350 and $293 \mathrm{~K}$ respectively, and water is taken as
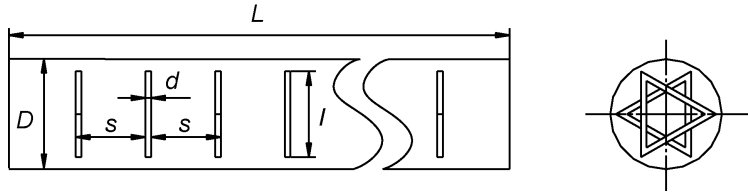

Figure 6 Computational model of a circular tube inserted with thin triangular pole arrangements. 
the fluid with its physical properties remaining constant.

Figures 7 and 8 illustrate the heat transfer and flow behaviors of the model. It is seen that the flow resistance in the heat-transfer-enhanced tube is greater than that in the bare tube because of fluid disturbance through the triangular pole arrangements. The average synergy angle $\alpha$ drops by $6^{\circ}-11^{\circ}$ within a range of the Reynolds number from 300 to 1800 . However, heat transfer is apparently enhanced, with a decrease in average synergy angle $\beta$ of $7^{\circ}$ within a range of the Reynolds number from 300 to 1800 . Therefore, controlling synergy angles $\alpha$ and $\beta$ in the flow field can intensify convective heat transfer and reduce flow resistance.

Figure 9 shows power consumption in the heat-transferenhanced tube. The one-way resistance and local resistance are greater in the heat-transfer-enhanced tube owing to the disturbance created by the triangular pole arrangements, and the kinetic loss is thus greater. As a result, synergy angle $\theta$ rises dramatically around $50^{\circ}$ when $R e=300-1800$. To reduce flow resistance, we can adjust the pole diameter and the arrangement interval, and then make the calculation judgment again using synergy angle $\theta$. It should be noted

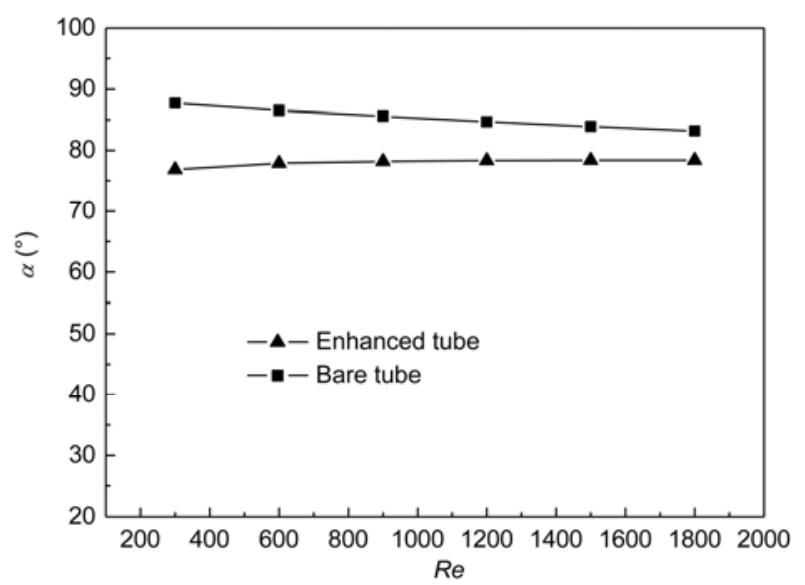

Figure 7 Synergy angle $\alpha$ versus Reynolds number for a bare tube and heat-transfer-enhanced tube.

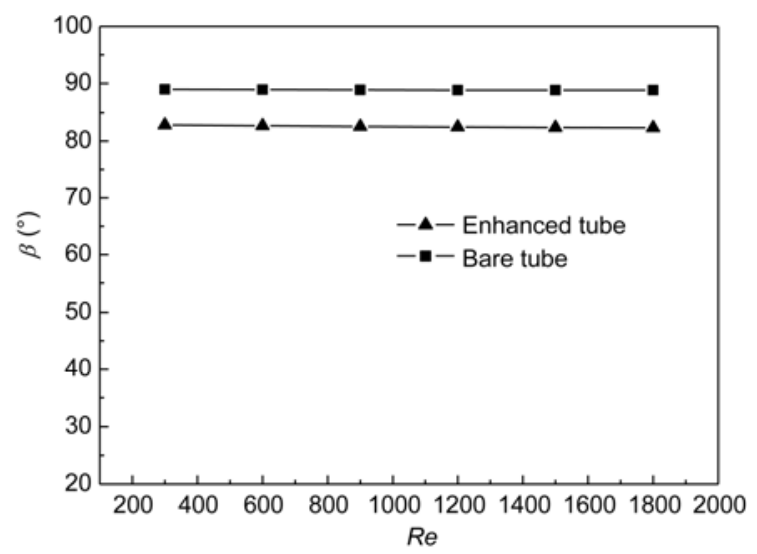

Figure 8 Synergy angle $\beta$ versus Reynolds number for a bare tube and heat-transfer-enhanced tube. that synergy angle $\theta$ provides more information than synergy angle $\alpha$ in evaluating the pressure drop in a heat transfer unit.

Figures 10 and 11 indicate the heat transfer ability and flow resistance of the heat-transfer-enhanced tube. It is seen that with an increase in the Reynolds number, the Nusselt number increases quickly and approaches 67 when $R e=$ 1800. This shows that the poles have the effect of enhancing convective heat transfer between the fluid and tube wall. On the other hand, the resistance coefficient rises by a factor of 5.5-8.3 within a range of the Reynolds number from 300 to 1800. Figure 12 shows the overall performance of heattransfer-enhanced tube. When $R e=1800$, the Nusselt number increases by a factor of 5.4, while the resistance coefficient increases by a factor of 8.3. Here PEC reaches 2.3 .

Figure 13 shows the temperature profiles of the fluid in the bare tube and heat-transfer-enhanced tube downstream at $z=465 \mathrm{~mm}$ with $R e=1800$. It is seen that compared with the case for the bare tube, the temperature in the core flow area of the heat-transfer-enhanced tube tends to be more uniform. This leads to a larger fluid temperature gradient near the tube wall, and it is beneficial to heat transfer. As the fluid region with uniform temperature becomes larger, the difference between the tube wall temperature and fluid

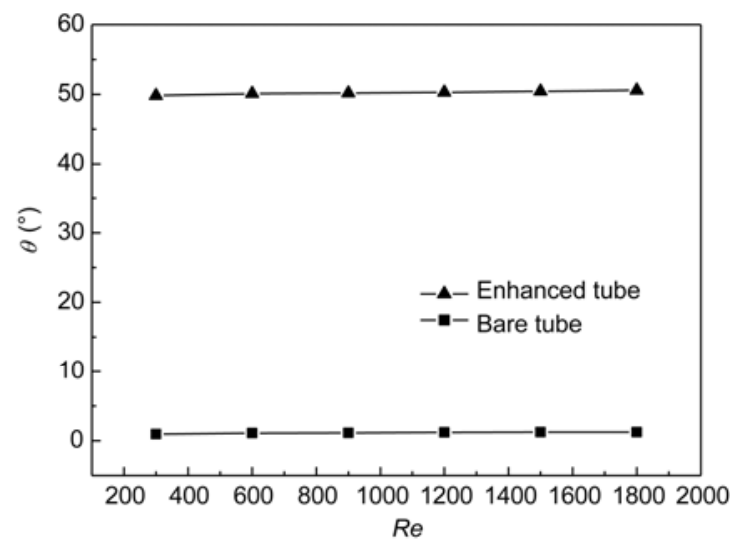

Figure 9 Synergy angle $\theta$ versus Reynolds number for a bare tube and heat-transfer-enhanced tube.

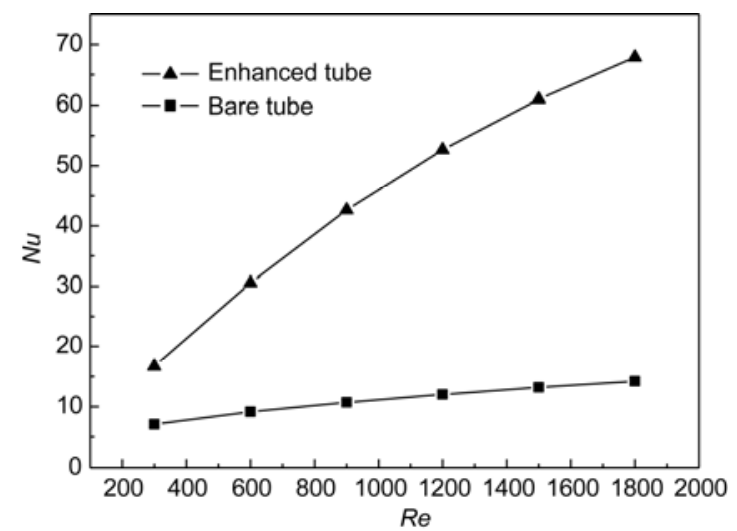

Figure 10 Nusselt number versus Reynolds number for a bare tube and heat-transfer-enhanced tube. 


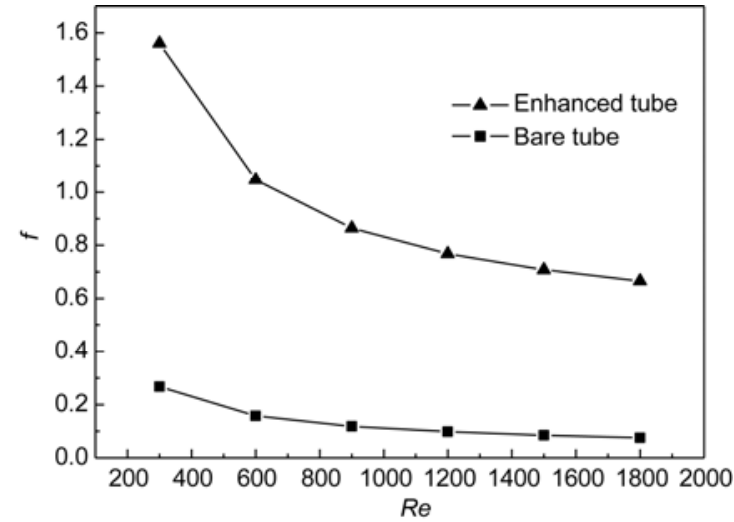

Figure 11 Resistance coefficient versus Reynolds number for a bare tube and heat-transfer-enhanced tube.

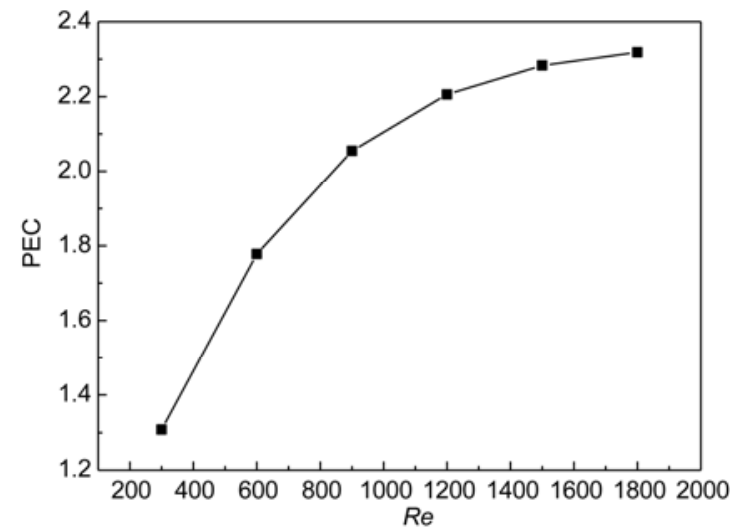

Figure 12 Value of PEC versus Reynolds number for a bare tube and heat-transfer-enhanced tube.

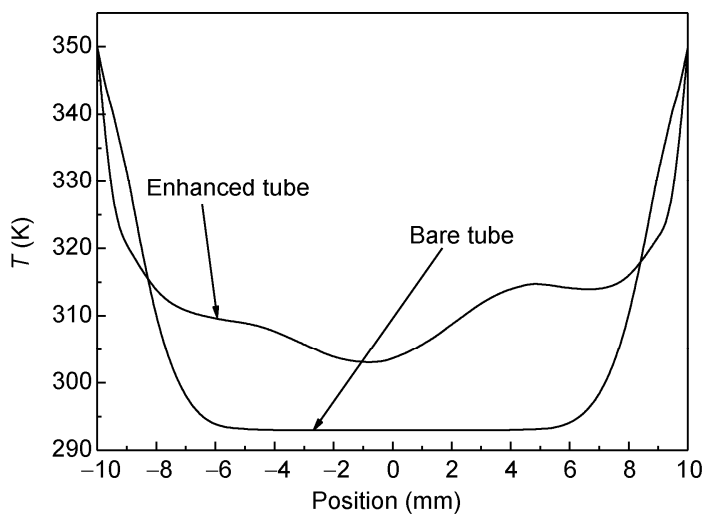

Figure 13 Fluid temperature profiles for a bare tube and heat-transferenhanced tube $(R e=1800, z=465 \mathrm{~mm})$.

mean temperature decreases, which means that thermal resistance between tube wall and fluid will be small at a certain boundary heat flux, and the transferring of heat will be better.

Figure 14 shows the variation in the Nusselt number with the Reynolds number for the bare tube and heattransfer-enhanced tube. From the figure, we find that the heat transferred by the heat-transfer-enhanced tube is $2-3.3$ times greater than that transferred by the bare tube in the range of $R e=300-1800$. In Figure 15, however, the calculation shows that the flow resistance of fluid is 4-9 times greater in the heat-transfer-enhanced tube than in the bare tube in the range of $R e=300-1800$. Figure 16 shows that EEC for the heat-transfer-enhanced tube is relatively small, and its maximum value is only 0.45 in the range of $R e=$ 300-1800. Therefore, the triangular pole arrangement is not recommended in engineering application. In general, we hope that the heat flux amplitude is close to or greater than the pressure difference amplitude, so that EEC can approach or exceed a value of 1 .

In summary, synergy angles $\alpha, \beta, \theta, \gamma$ and $\eta$ are formed from the velocity vector $\boldsymbol{U}$, velocity gradient $\nabla u$, temperature gradient $\nabla T$ and pressure gradient $-\nabla p$, and they denote the relation between field synergy and convective heat transfer enhancement. Mathematical formulas were established to describe the multi-field synergy principle. One can then use the principle to evaluate the performance of heat transfer units, and guide the design of heat exchangers.

\section{Conclusions}

Synergy angles $\alpha, \beta, \theta, \gamma$ and $\eta$ reflect multi-field synergy

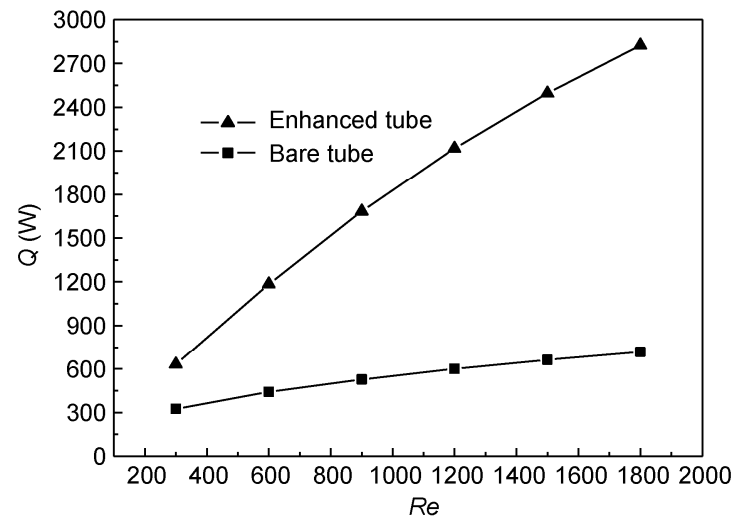

Figure 14 Nusselt number versus Reynolds number for a bare tube and heat-transfer-enhanced tube.

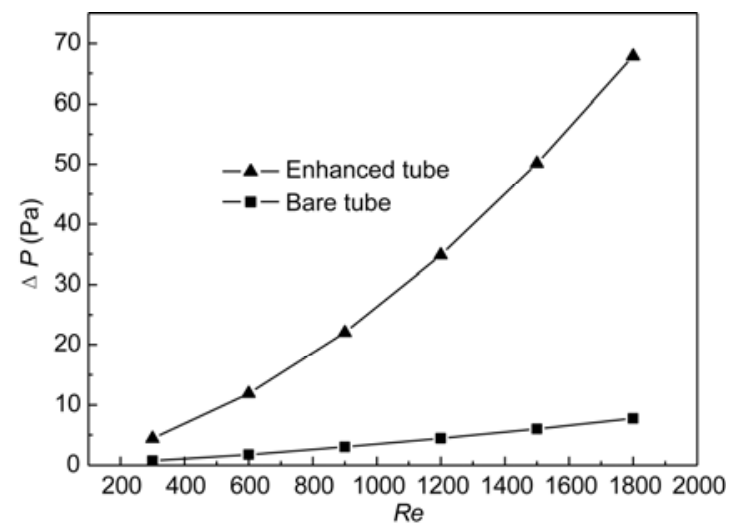

Figure 15 Pressure difference versus Reynolds number for a bare tube and heat-transfer-enhanced tube. 


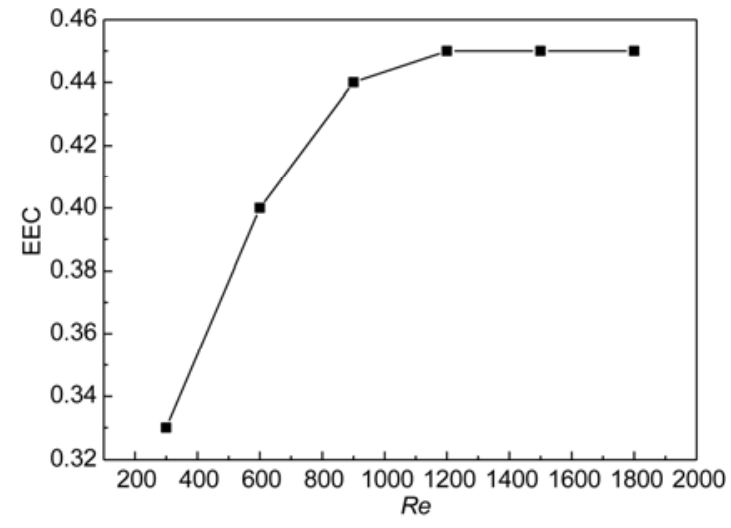

Figure 16 Value of EEC versus Reynolds number for a bare tube and heat-transfer-enhanced tube.

regularity in convective heat transfer. The higher the synergy degree is, the more effective the heat transfer enhancement will be. The vector mode values $|\nabla T|,|\nabla u|$ and $|\nabla p|$ describe the intensity of heat transfer and flow. The larger the $|\nabla T|$ value in the boundary layer is, the greater the heat transfer will be; the smaller the $|\nabla u|$ or $|\nabla p|$ value is, the smaller the flow resistance will be.

The performance of a heat transfer unit can be evaluated with synergy angles $\beta$ and $\theta$, and also with the efficiency evaluation criterion EEC, which represents the overall effect of heat transfer enhancement. The smaller the angles $\beta$ and $\theta$ are, the smaller the heat resistance and the flow resistance will be; the greater the EEC value is, the better the comprehensive performance of the heat-transfer-enhanced tube will be.

From calculations for the heat-transfer-enhanced tube with inserts of triangular arrangements of tiny poles, we find that the average synergy angle $\beta$ decreases from approximately $90^{\circ}$ for the bare tube to about $83^{\circ}$ for the heattransfer-enhanced tube, which indicates an obvious enhancement in heat transfer; the average synergy angle $\theta$ increases from approximately $0^{\circ}$ for the bare tube to about $50^{\circ}$ for the heat-transfer-enhanced tube, which indicates a quick increase in flow resistance; when $R e=1800$, PEC reaches 2.3, but EEC is about 0.45 . The latter finding demonstrates the overall relation between income and cost in energy utilization.

This work was supported by the National Natural Science Foundation of China (51036003, 51021065) and the National Basic Research Program of China (2007CB206903).

1 Webb R L. Principles of Enhanced Heat Transfer. New York: Wiley, 1994
2 Bergles A E. ExHFT for fourth generation heat transfer technology. Exp Therm Fluid Sci, 2002, 26: 335-344

3 Guo Z Y, Li D Y, Wang B X. A novel concept for convective heat transfer enhancement. Int J Heat Mass Transfer, 1998, 41: 2221-2225

4 Zhao T S, Song Y J. Forced convection in a porous medium heated by permeable wall perpendicular to flow direction: Analyses and measurements. Int J Heat Mass Transfer, 2001, 44: 1031-1037

5 Tao W Q, Guo Z Y, Wang B X. Field synergy principle for enhancing convective heat transfer-Its extension and numerical verification. Int J Heat Mass Transfer, 2002, 45: 3849-3856

6 Tao W Q, He Y L, Wang Q W, et al. A unified analysis on enhancing single phase convective heat transfer with field synergy principle. Int J Heat Mass Transfer, 2002, 45: 4871-4879

7 Shen S, Liu W, Tao W Q. Analysis of field synergy on natural convective heat transfer in porous media. Int Comm Heat Mass Transfer, 2003, 30: 1081-1090

8 Tao W Q, He Y L. Application of the field synergy principle in developing new type heat transfer enhanced surfaces. J Enhanc Heat Transfer, 2004, 11: 433-449

9 Qu Z G, Tao W Q, He Y L. Three-dimensional numerical simulation on laminar heat transfer and fluid flow characteristics of strip fin surface with X-arrangement of strips. J Heat Transfer, 2004, 126: 697707

10 Cheng Y P, Qu Z G, Tao W Q, et al. Numerical design of efficient slotted fin surface based on the field synergy principle. Numer Heat Transfer A, 2004, 45: 517-538

11 Chen W L, Guo Z Y, Chen C K. A numerical study on the flow over a novel tube for heat transfer enhancement with linear eddy-viscosity model. Int J Heat Mass Transfer, 2004, 47: 3431-3439

12 Guo Z Y, Tao W Q, Shah R K. The field synergy (coordination) principle and its applications in enhancing single phase convective heat transfer. Int J Heat Mass Transfer, 2005, 48: 1797-1807

13 Chen C K, Yen T Z, Yang Y T. Lattice Boltzmann method simulation of backward-facing step on convective heat transfer with field synergy principle. Int J Heat Mass Transfer, 2006, 49: 1195-1204

14 Ma L D, Li Z Y, Tao W Q. Experimental verification of the flied synergy principle. Int Comm Heat Mass Transfer, 2007, 34: 269-276

15 Cai R X, Gou C H. Discussion of the convective heat transfer and field synergy principle. Int J Heat Mass Transfer, 2007, 50: 51685176

16 Cheng Y P, Lee T S, Low H T. Numerical simulation of conjugate heat transfer in electronic cooling and analysis based on field synergy principle. Appl Therm Eng, 2008, 28: 1826-1833

17 Chen Q, Ren J X, Meng J A. Field synergy equation for turbulent heat transfer and its application. Int J Heat Mass Transfer, 2007, 50: 5334-5339

18 Zeng M, Tao W Q. Numerical verification of the field synergy principle for turbulent flow. J Enhanc Heat Transfer, 2004, 11: 451-457

19 Liu W, Liu Z C, Huang S Y. Physical quantity synergy in the field of turbulent heat transfer and its analysis for heat transfer enhancement. Chin Sci Bull, 2010, 55: 2589-2597

20 Liu W, Liu Z C, Guo Z Y. Physical quantity synergy in laminar flow field of convective heat transfer and analysis of heat transfer enhancement. Chin Sci Bull, 2009, 54: 3579-3586

21 Liu W, Liu Z C, Ming T Z, et al. Physical quantity synergy in laminar flow field and its application in heat transfer enhancement. Int J Heat Mass Transfer, 2009, 52: 4669-4672

22 Webb R L, Eckert E R. Applications of rough surfaces to heat exchanger design. Int J Heat Mass Transfer, 1972, 15: 1647-1658

23 Webb R L. Performance evaluation criteria for use of enhanced heat transfer surfaces in heat exchanger design. Int J Heat Mass Transfer, 1981, 24: 715-726

24 Patankar S V. Numerical Heat Transfer and Fluid Flow. New York: McGraw-Hill, 1980

Open Access This article is distributed under the terms of the Creative Commons Attribution License which permits any use, distribution, and reproduction in any medium, provided the original author(s) and source are credited. 